International Journal of Quantum Information

Vol. 3, No. 4 (2005) 649-654

(C) World Scientific Publishing Company

\title{
FEASIBILITY OF THE INTERLOCK PROTOCOL AGAINST MAN-IN-THE-MIDDLE ATTACKS ON QUANTUM CRYPTOGRAPHY
}

\author{
KARL SVOZIL \\ Institut für Theoretische Physik, University of Technology Vienna, \\ Wiedner Hauptstraße 8-10/136, A-1040 Vienna, Austria \\ svozil@tuwien.ac.at \\ Received 12 March 2005 \\ Revised 6 April 2005
}

\begin{abstract}
If an eavesdropper succeeds in compromising the quantum as well as the classical channels and mimics the receiver "Bob" for the sender "Alice" and vice versa, one defence strategy is the successive, temporally interlocked partial transmission of the entire encrypted message.
\end{abstract}

Keywords: Quantum information; quantum communication; quantum cryptography.

Ah, love, let us be true To one another! for the world, which seems To lie before us like a land of dreams, So various, so beautiful, so new, Hath really neither joy, nor love, nor light, Nor certitude, nor peace, nor help for pain; And we are here as on a darkling plain Swept with confused alarms of struggle and flight, Where ignorant armies clash by night.

from Dover Beach by Matthew Arnold (1822-1888)

Since the introduction of conjugate coding by Wiesner in the $1970 \mathrm{~s},{ }^{1}$ quantum cryptography ${ }^{2-7}$ has developed into an increasingly active area of applied research and technology, as well as a powerful tool to exploit the quantum. From the first realization at IBM's Yorktown Heights laboratory ${ }^{5}$ to its implementation across Lake Geneva ${ }^{6}$ and various other spots around the world, including the Boston metropolitan area ${ }^{8}$ and the Viennese sewage system, ${ }^{7}$ quantum cryptographic techniques are among the finest experiments ever performed.

It is often argued that, as long as an eavesdropper "Eve" can only measure the communicated quanta, the principles of physics and thus Nature itself protect the secrecy. Several proofs of the unconditional security of quantum cryptographic protocols have been published (e.g. Refs. 9 and 10) supporting this line of reasoning. Few of them (e.g. Refs. 11-13) discuss man-in-the-middle attacks as a feasible 
cryptanalytic method. Alas, while we have no intention of challenging these claims under the assumptions made, not all eavesdroppers may stick to these rules. It is not entirely unjustified to suspect that if Eve is capable of compromising the quantum channel, she may also be capable of compromising the classical channel. Indeed, the possible interception of classical communication is often taken as the very reason to propose an additional quantum channel and quantum cryptographic protocols in general. As either the security of the classical channel or classical authentication is required for secure quantum cryptographic protocols, it is not totally unjustified to state that quantum cryptography cannot be considered qualitatively safer than classical cryptography. It is nevertheless interesting to investigate the possible defence strategies against cryptanalytic attacks. In what follows, after a brief review of man-in-the-middle attacks, we shall concentrate on the interlock protocol as an additional defence measure capable of reducing authentication. Temporal interlocks have not yet been discussed in the quantum cryptographic context.

If Eve is able to intercept the classical as well as the quantum channels between the sender Alice and the receiver Bob, a rather straightforward man-in-the-middle attack can be launched ${ }^{3,5,14^{-16}}$ (see also the middleperson attack discussed in Ref. 17), which has been discussed already in the BB84 paper ${ }^{3}$ but seems to have gone unnoticed in the public perception of quantum cryptography and is also not mentioned in many security proofs.

Let us thus shortly review man-in-the-middle attacks. In the configuration discussed, we assume that a message is transferred from Alice to Bob, and hence the scheme is forward directed. In the case of key generation, like in the BB84 protocol, Eve's task is simpler, as no indirect communication between Ann and Bob via Eve needs to take place; Eve may communicate with each one of them separately. Eve's part consists of three main phases. The three phases are schematically represented in Fig. 1. Very similar procedures hold for non-information-directed protocols used to generate keys.

(i) In the first phase, Eve makes Alice believe that Alice is communicating with Bob while she is actually communicating with Eve. This, of course, can only be achieved by intercepting both classical and quantum channels. Thereby,

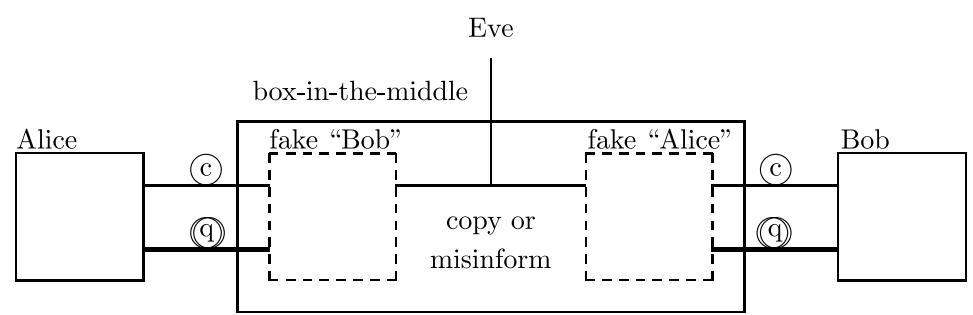

Fig. 1. Scheme of a man-in-the-middle attack. 
Alice's message which was intended for Bob can be deciphered and re-encoded classically.

(ii) In the second phase, Eve processes the classical message to suit her goals. For instance, the classical code can either be copied or modified — or, a totally new message, completely unrelated to Alice's original message, may be prepared for the next phase.

(iii) In the third phase, Eve invokes the same type of protocol as in the first phase to re-encode the message prepared for Bob in the second phase, and then sends it to Bob.

This attack is based on the problem of proper authenticating a legal sender and receiver. Substitution of the classical channel by another quantum channel cannot solve the authentication problem.

For a similar classical example, take a message which is encoded into two binary substrings $x$ and $y$ and transmitted over two separate classical channels. Suppose a plain message which is to be transmitted secretly is coded into a binary sequence $y=y_{1} y_{2} \cdots$, with $y_{i} \in\{0,1\}$ in any usual, unencrypted form. Let the first channel convey an arbitrary random binary sequence $x=x_{1} x_{2} \cdots$, with $x_{i} \in\{0,1\}$. In the second channel, an encrypted sequence $z=z_{1} z_{2} \cdots$, which is the sum modulus two (the bitwise exclusive-or) $z_{i}=\left(x_{i}+y_{i}\right) \bmod 2$ of $x$ and $y$ is transmitted. In such cases, an eavesdropper can only decipher the message and recover $y$ if both channels are intercepted. The only difference between this scenario and the quantum one is that, because of the no-cloning theorem, copying of a generic quantum bit is not allowed, and hence $x$ and $z$, if at least one of them is communicated via a quantum channel, cannot pass an eavesdropper unaltered. But these obstacles can be circumvented by completely absorbing and re-emitting a quantum code, as described above.

Usually, classical authentication is proposed as a defence against man-in-themiddle attacks, resulting in a quantum cryptographic scheme which relies on classical cryptology. We shall consider here another strategy based on temporal synchronization. This counter strategy cannot be directly applied to key generation, as in this case no message is transferred between Alice and Bob. But it may be helpful in an encoded information transfer between Alice and Bob.

Suppose Eve merely intends to copy the message and not to misinform. Then, if the quantum protocol is not instantaneous, only after completion of a sequence can she start transmitting a copy of the message to Bob. As a result, there is a time lag during which Eve completes receiving Alice's message and retransmits it to Bob.

There are three shortcomings of a straightforward defence strategy based on time synchronization: (i) Time lags are intrinsic features of many quantum cryptographic protocols, which do not result in instantaneous codes. On the other hand, the bigger the time lags for decryption, the easier it might be to detect eavesdropping through man-in-the-middle attacks. (ii) Proper time synchronization between Alice and Bob would require a secure classical channel, which was excluded by the assumptions. 
However, for practical purposes, synchronization via satellite-based GPS systems may be a feasible classical synchronization channel difficult to actively intercept. The problem remains to securely negotiate the onset of the protocol, as already in this phase Eve may negotiate with the respective partners. (iii) In the case of misinformation (Eve misinforming Bob irrespective of the information she receives from Alice), no copy of Alice's original message is required, and there need not be any time lag at all.

A refined strategy against classical man-in-the-middle attacks is the interlock protocol proposed by Rivest and Shamir ${ }^{18}$ as well as timed-release cryptography by May. ${ }^{19,20}$ The interlock protocol can be divided into four phases depicted in Fig. 2:

(i) Alice encrypts her message by a code which is not instantaneously decodable. Conversely, Bob encrypts his message by a code which is not instantaneous. (Part of this message may, for instance, consist of a classic authentication.)

(ii) Alice sends only a fraction of her encrypted message to Bob, and Bob sends only a fraction of his encrypted message to Alice.

(iii) Upon receiving Bob's partial message, Alice sends the rest of her encrypted message to Bob; and upon receiving Alice's partial message, Bob sends the rest of his message to Alice. (A generalization to the splitting of the encoded message into more than two packets is straightforward.)

(iv) Alice and Bob put the two parts of the received encrypted messages together.

The advantage of the interlock over unbroken transmission is that until phase three, Eve possesses only part of Alice's and Bob's encoded messages and is unable to decode them for partial retransmission. Only in the final phase can the entire messages be recovered. Thus, if time synchronization is maintained and observed, Eve has no other chance than to fake the first messages exchanged by Alice and Bob.

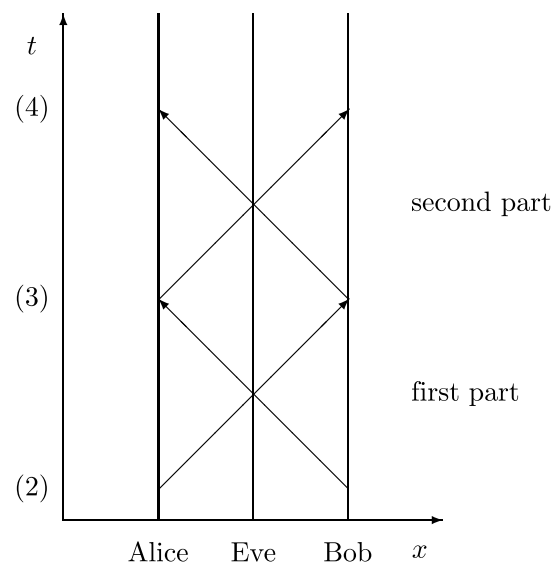

Fig. 2. Scheme of the interlock protocol. Numbers in brackets indicate the phases mentioned in the text. 
The difference between classical and quantum interlock protocols resides in the quantum no-cloning theorem and in complementarity: in the quantum case, copying or measurement of the encoded messages exchanged by Alice and Bob is impossible and would result in a randomization of these messages. Absorption, decoding and the reemission of the re-encoded total message is still possible, resulting in a time lag which could be monitored. Alternatively, Eve could fake the packets of the first complete message exchanged (thus misinforming Alice and Bob), and could subsequently copy and retransmit the $(n-1)$-th packet in the duped communication in the time slot reserved for a reception of the $n$th packet.

Additional authentication of Alice and Bob would make it impossible for Eve to fake the first messages exchanged. Thus, if it were be possible, for example, by using satellite-based GPS systems, to synchronize Alice's and Bob's clocks, then the interlock protocol could be applied as an additional resource requiring classical authentication to rule out a quantum man-in-the-middle attack by demanding to keep the interlocked phases in temporal order. As mentioned above, the ability to synchronize clocks represents an additional authentication resource which is not present in the quantum cryptographic protocols so far discussed or realized.

In summary, sending interlocked partial sequences may be a practically feasible defence against man-in-the-middle attacks on quantum encrypted message transfers. It cannot be applied to quantum key generation. Classical authentication methods remain indispensable to secure the legal identity of the sender and the receiver. With respect to man-in-the-middle attacks, quantum cryptography shares the same vulnerability and cannot be considered principally safer than classical cryptography. We therefore agree with the position that quantum cryptography should be perceived more as a secret key expansion ${ }^{2,3}$ or, in other words, a secret key growing technique ${ }^{16}$ rather than as a secret key generation scheme. We believe that, instead of glorious claims, the vulnerabilities of quantum cryptography need to be stated clearly for a proper comprehension of the general public and also for the risk portfolio management of potential clients.

\section{References}

1. S. Wiesner, Sigact News 15, 78 (1983) [manuscript written circa 1970].

2. C. H. Bennett, G. Brassard, S. Breidbart and S. Wiesner, Quantum cryptography, or unforgable subway tokens, in Advances in Cryptography: Proc. Crypto '82, August, 1982, eds. D. Chaum, R. L. Rivest and A. T. Sherman (Plenum Press, New York, 1982), pp. 267-275.

3. C. H. Bennett and G. Brassard, Quantum cryptography: Public key distribution and coin tossing, in Proc. IEEE Int. Conf. Computers, Systems, and Signal Processing, Bangalore, India, 10-12 December, 1984 (IEEE Computer Society Press, 1984), pp. $175-179$.

4. A. Ekert, Phys. Rev. Lett. 67, 661 (1991).

5. C. H. Bennett, F. Bessette, G. Brassard, L. Salvail and J. Smolin, J. Cryptol. 5, 3 (1992).

6. N. Gisin, G. Ribordy, W. Tittel and H. Zbinden, Rev. Mod. Phys. 74, 145 (2002). 
7. A. Poppe et al., Practical quantum key distribution with polarization-entangled photons, Opt. Express 12, 3865 (2004), quant-ph/0404115.

8. C. Elliott et al., Current status of the DARPA quantum network (2005), quant$\mathrm{ph} / 0503058$.

9. P. W. Shor and J. Preskill, Phys. Rev. Lett. 85, 441 (2000), quant-ph/0003004.

10. D. Mayers, J. ACM 48, 351 (2001), quant-ph/9802025.

11. N. Lütkenhaus, Phys. Rev. A 59, 3301 (1999), quant-ph/9806008.

12. D. Bruß and N. Lütkenhaus, Appl. Algebr. Eng. Comm. 10, 383 (2000), quantph/9901061.

13. G. Gilbert and M. Hamrick, Practical quantum cryptography: A comprehensive analysis (Part 1) (2000), quant-ph/0009027.

14. D. R. Kuhn, Vulnerabilities in quantum key distribution protocols (2003), quant$\mathrm{ph} / 0305076$.

15. K. G. Paterson, F. Piper and R. Schack, Why quantum cryptography? (2004), quant-ph/0406147.

16. M. Peev et al., A novel protocol-authentication algorithm ruling out a man-inthe-middle attack in quantum cryptography, Int. J. Quant. Inform. 3, 225 (2005), quant-ph/0407131.

17. S. Bengio, G. Brassard, Y. G. Desmedt, C. Goutier and J.-J. Quisquater, J. Cryptol. 4, 175 (1991).

18. R. L. Rivest and A. Shamir, Commun. ACM 27, 393 (1984).

19. T. C. May, Timed-release crypto (1993), unpublished.

20. R. L. Rivest, A. Shamir and D. A. Wagner, Time-lock puzzles and timed-release cryptography, LCS technical memo MIT/LCS/TR-684 (1996). 